\title{
Animal Models of Virus-Induced Neurobehavioral Sequelae: Recent Advances, Methodological Issues, and Future Prospects
}

\author{
Marco Bortolato and Sean C. Godar \\ Department of Pharmacology and Pharmaceutical Sciences, School of Pharmacy, University of Southern California, \\ Los Angeles, CA 90089, USA \\ Correspondence should be addressed to Marco Bortolato, bortolat@usc.edu
}

Received 15 August 2009; Revised 14 November 2009; Accepted 9 March 2010

Academic Editor: Marylou V. Solbrig

Copyright ( 2010 M. Bortolato and S. C. Godar. This is an open access article distributed under the Creative Commons Attribution License, which permits unrestricted use, distribution, and reproduction in any medium, provided the original work is properly cited.

\begin{abstract}
Converging lines of clinical and epidemiological evidence suggest that viral infections in early developmental stages may be a causal factor in neuropsychiatric disorders such as schizophrenia, bipolar disorder, and autism-spectrum disorders. This etiological link, however, remains controversial in view of the lack of consistent and reproducible associations between viruses and mental illness. Animal models of virus-induced neurobehavioral disturbances afford powerful tools to test etiological hypotheses and explore pathophysiological mechanisms. Prenatal or neonatal inoculations of neurotropic agents (such as herpes-, influenza-, and retroviruses) in rodents result in a broad spectrum of long-term alterations reminiscent of psychiatric abnormalities. Nevertheless, the complexity of these sequelae often poses methodological and interpretational challenges and thwarts their characterization. The recent conceptual advancements in psychiatric nosology and behavioral science may help determine new heuristic criteria to enhance the translational value of these models. A particularly critical issue is the identification of intermediate phenotypes, defined as quantifiable factors representing single neurochemical, neuropsychological, or neuroanatomical aspects of a diagnostic category. In this paper, we examine how the employment of these novel concepts may lead to new methodological refinements in the study of virus-induced neurobehavioral sequelae through animal models.
\end{abstract}

\section{The Role of Viruses in Psychiatric Disorders}

The implication of viruses in the pathogenesis of neuropsychiatric disorders has been posited since the foundations of modern psychiatry. Few years after the outbreak of the 1918 flu pandemic, the nosographic analogies between postinfluenza encephalitic manifestations and psychotic traits prompted Menninger [1] to conjecture an infective etiology for some subtypes of schizophrenia. Although this hypothesis sank into relative obsolescence during the following decades, the identification of lentiviruses rekindled enthusiasm for the viral theory of mental disorders and laid the foundations of psychovirology as a novel discipline [2]. Over the last 25 years, numerous studies have shown that viral infections of the central nervous system are potentially conducive to profound behavioral and cognitive perturbations. Typical outcomes of viral encephalitis, for instance, encompass impairments in sensory reactivity and information processing, emotional lability, and sleep disturbances. Notably, the severity of some postencephalitic manifestations is often correlated to the degree of morphological and functional damage induced by both the viral infection and/or the ensuing immune response [3]. A detailed analysis of these phenomena is beyond the scope of this paper and can be found elsewhere $[4,5]$.

Neuropsychiatric sequelae of overt infectious processes, while accounting for few documented cases of mental illness, offer a compelling theoretical platform to postulate the role of viruses in the pathophysiology of schizophrenia and other neurodevelopmental syndromes. According to the prevalent theories, the pathogenesis of these disorders may be initiated or contributed by early exposure to lowvirulence, neurotropic viruses. The resulting infection, albeit subclinical, may interfere with key neurogenetic processes and lead to insidious, potentially ingravescent functional impairments. Specifically, this neuropathic process has been 
shown to reflect the combination of the insults mediated by the cytopathic and mutagenic effects of the virus [6], as well as the immune response [7-10]. Furthermore, the chronic enactment of compensatory, neuroprotective responses may also contribute to the pathogenesis of these disorders. For example, certain clusters of psychotic manifestations may result from the outcome of the hippocampal dysregulation by virus-mediated and endogenous anticonvulsant mechanisms [11]. The confluence of these processes with neurodevelopmental mechanisms and other factors of vulnerability (both genetic and environmental) may result in severe neurochemical imbalances, brain architectural irregularities, and mental derangement.

The implication of viruses in mental disorders is supported by several lines of epidemiological evidence. Prenatal or perinatal viral infections are risk factors for schizophrenia and autism-spectrum disorders [12, 13]. Numerous epidemiological surveys have also documented a birth excess of schizophrenia and bipolar patients in winter and spring [1417] or seasonal correlations between incidence of autismspectrum disorders and viral outbreaks [18-22].

The viral hypothesis of mental disorders is also corroborated by clinical evidence. Several investigations have shown the presence of viral antigens and antibodies in plasma or post-mortem samples of mental patients [23-25]. In particular, these studies have revealed that schizophrenia may be associated with several families of viruses, including herpes viruses [26-30], orthomyxoviruses [31-35], and retroviruses $[36,37]$. It is worth noting that most of these agents have also been associated with autism-spectrum disorders [38, 39], bipolar disorder [40-42], and other psychiatric conditions [43].

However, there remain numerous inconsistencies across different clinical studies concerning the role of viruses in psychiatric disorders. The lack of reproducible associations may be contingent on different parameters related to the virus (rarity, reproductive cycle, virulence, latency), host (age at the time of infection, genetic vulnerability, immune responsiveness) and infective process (modality of transmission, duration and severity of the infection, patterns of viral replication and distribution within the host, viral interaction with other infective agents and environmental factors). Other discrepancies may reflect methodological limitations in the design and execution of the studies, including imprecise diagnostic assessments, poor sensitivity and specificity of survey criteria, insufficient number of subjects, low statistical power, and lack of adequate controls.

\section{Animal Models of Virus-Induced Neurobehavioral Sequelae}

2.1. Choice of Animal. Animal models provide a powerful tool to explore the biological substrates of virus-induced neuropsychiatric sequelae in a controlled experimental setting. The best target to address specific pathophysiological hypotheses is afforded by mammalian species, and in particular nonhuman primates, in view of their relatively high degree of anatomical and phylogenetic continuity with humans. Ethical considerations, however, dictate that experimentation on monkeys should be strictly limited to exceptional circumstances, where no viable alternatives are available, such as the research on simian immunodeficiency virus as a neuroAIDS model. Rodents offer an attractive compromise for research on viral neurobehavioral disorders, by virtue of their advantageous characteristics, including small size, short reproductive cycle, and cost-effectiveness. The inherent differences between humans and rodent species in the key factors of pathogenesis of virus-induced sequelae-such as neurodevelopmental mechanisms and immune reactivity - are an important limiting factor in the application of these models. These shortcomings notwithstanding, rodent models have a high level of validity, which is warranted by common physiological, neurobiological, and ethological characteristics. Indeed, investigations on mice (Mus musculus) and rats (Rattus norvegicus) have become the richest source of knowledge on the neural substrates and molecular underpinnings of post-infectious behavioral dysregulations. These models have been instrumental to the acquisition of a large body of information on virus-induced neurobehavioral alterations.

A thorough overview of the body of evidence on the specific virus-induced neurobehavioral alterations is covered in the excellent reviews by Tomonaga [5] and Weed and Gold [44]. In the following sections, we will focus on the key conceptual issues and advances in rodent models of virus-induced neurobehavioral disorders and indicate new potential experimental directions to reduce confounding factors in the analysis of these abnormalities.

2.2. Validity. Rodent models of virus-induced neurobehavioral alterations are a striking example of homologous models, aimed at reproducing the etiology, pathophysiology and symptomatic presentation of psychiatric disorders. In substantial agreement with the classical criteria of validity for animal models [45], the degree of isomorphism between virus-induced sequelae and the corresponding psychiatric should be assessed at the following four levels:

(i) etiological validity, based on the epidemiological and/or clinical relevance of the pathogen agents to the targeted disorder;

(ii) face validity, describing the analogies between the virus-induced behavioral manifestations of the animal model and the signs and symptoms of the targeted psychiatric disorder;

(iii) predictive validity, signifying the responsiveness of the model to clinically efficacious treatments (such as antipsychotic agents for schizophrenia etc.);

(iv) construct validity, representing the pathophysiological congruence between animal and human neurobiological alterations.

A prominent case of fulfillment of the above standards is illustrated by the outcomes of prenatal and/or early postnatal exposure to influenza virus as a model of schizophrenia:

(i) the etiological validity of the model is strongly supported by the aforementioned epidemiological 
association between influenza and psychotic disorders;

(ii) the face validity of the model is ensured by the resemblance between the symptoms of schizophrenia and the array of behavioral changes observed in the animals, including alterations in exploratory activity, social behavior, emotional reactivity, gating, sleep patterns, working, and spatial memory [46-51];

(iii) the predictive validity of the model is based on its responsiveness to atypical antipsychotic agents [50];

(iv) the construct validity of the model is warranted by the implication of some of the key areas in the pathophysiology of schizophrenia, such as neocortex and hippocampus $[52,53]$, as well as by the demonstration of alterations in line with those shown in these disorders, such as alterations of pyramidal neurons, glia, and myelination processes [52-59].

Another example is represented by rodents subjected to early HSV inoculation, which exhibit an array of schizophrenia-related deficits, including preattentional and cognitive impairments [60-63] and alterations of the serotonergic system and hippocampus $[64,65]$.

Although the adoption of stringent validity criteria ensures high sensitivity and specificity of preclinical findings, caution should be exercised in their utilization. In particular, over-reliance on the use of reference compounds as gold standards for predictive validity-such as conventional antipsychotic agents for models of virus-induced psychotic disorders-has been shown to impoverish the translational potential of numerous models $[66,67]$. Furthermore, the etiological validity of these models is inherently tempered by the impossibility to reproduce specific virus-host interactions. In view of these considerations, several lines of research have recently focused on the identification of relevant behavioral manifestations and pathophysiological alterations induced by neurotropic viruses, irrespective of their proven implication of human pathology. This concept is clearly exemplified by experimentation on bornadisease virus (BDV), a single-stranded RNA virus whose association with in behavioral disorders is well-established in animals, yet still highly controversial in humans $[68,69]$. BDV infection triggers a broad spectrum of abnormalities in animals, which encompass emotional impairments, aggressiveness, stereotyped behavior, and cognitive deficits $[70,71]$. These alterations are supported by neurochemical changes in dopamine and neurotrophin signaling [71-76]. These and other studies of viral effects on neurotransmitter, neurohormonal, neurotrophic systems, and inflammatory mediators have represented a useful template to study the mechanisms of virus- and immune-mediated behavioral disorders, generalizable to other viral and pathogen agents.

2.3. Intermediate Phenotypes and Endophenotypes. A critical complication posed by models of psychiatric disorders is based on the absence of biomarkers and other quantitative indices in the current criteria of classification outlined in the Diagnostic and Statistical Manual of Mental Disorders
(DMS) IV TR. This absence results in a mismatch between the diagnostic and the pathophysiological perspectives. Nosologic categories such as schizophrenia, for example, are merely based on limited symptomatic and diagnostic criteria and may be broad rubrics encompassing a number of heterogeneous disorders with overlapping semeiological features. Conversely, conditions with similar etiology and pathophysiology may be set apart by artificial diagnostic criteria. In this perspective, viral sequelae may only represent specific subsets of mental disorders.

The acknowledgement of this scenario has fostered a lively academic debate on the reorganization of translational principles in psychiatry and reiterated the necessity of incorporating pathophysiological criteria in the classification of mental disorders [67]. This discussion has led to the implementation of quantitative, pathophysiological, and dimensional criteria in the new conceptual guidelines for the next edition of the DSM (DSM-V) [77-79].

An important corollary of this theoretical reorganization of translational science has been the development of the concept of intermediate phenotypes. These quantifiable factors represent neuroanatomical, biochemical, neurophysiological, neuropsychological, or cognitive traits, reflecting a simpler architecture than the whole array of abnormalities featured in a diagnostic category [80]. An important variant of this notion is that of endophenotype, defined as a heritable intermediate phenotype which, albeit not inherently pathological, is an element of vulnerability to mental disorders $[81,82]$. The validation of a biological characteristic as an endophenotype is dictated by a set of criteria that reflect and emphasize its genetic nature, including cosegregation in nonaffected relatives of the probands $[83,84]$.

A typical example of a well-established neuropsychological endophenotype is the prepulse inhibition (PPI) of the startle reflex. PPI is the reduction in startle response elicited by a strong sensory stimulus that occurs when the latter is preceded by a weaker signal [85]. This parameter is considered a highly dependable index for the measurement of gating function, and its deficits are typically observed in schizophrenia, bipolar disorder, autism, and other disorders [86]. Notably, PPI deficits are not only featured by mental patients, but also by their nonaffected relatives $[87,88]$.

Although the manifestations of endophenotypes may become apparent only in correspondence of specific environmental contingencies, they are always active and independent from the symptomatic fluctuations of the associated disorder. Furthermore, endophenotypes are generally not pathognomonic of specific mental disorders, but they are often featured in different diagnostic categories, probably signifying common pathophysiological mechanisms between disorders.

Endophenotypes have recently garnered increasing popularity in psychiatry, as they offer a suitable operational appendix to the body of knowledge derived from the characterization of human genome and generation of multiple lines of transgenic rodents. Furthermore, this strategy may be vital for the progress in our understanding of neurodevelopmental disorders [89-93]. The dissection of complex behavioral syndromes into more elementary "building blocks"— such as 
quantifiable responses related to specific neuropsychological traits-is more amenable to effective translational strategies and may lead to a profitable exchange of information between human and animal studies and the identification of common indices in mental patients and animal models $[67,94,95]$.

Analogous considerations can be applied to the case of virus-derived neurobehavioral abnormalities. In particular, the identification of intermediate phenotypes in virusbased animal models is critical to simplify the mapping of their abnormalities, elucidate pathophysiological links, and enhance the translational value of these experimental preparations. In parallel with the criteria postulated for the definition of endophenotypes in behavioral genetics, virusdependent intermediate phenotypes could be theorized as outcomes of viral (or immune) action on specific neurobiological substrates. These neurochemical, morphological, or psychological traits should reflect specific factors of vulnerability to a neurodevelopmental disorder, but not overt pathological manifestations. Accordingly, they should also be observed in mental patients as well as in nonaffected individuals with an overlapping history of early viral infection (or at least analogous antibody panels, signifying similar antigenic history).

The identification of these targets in humans, however, is extremely arduous, in view of the high variability of natural conditions affecting the infectious process and its outcomes. Thus, high-quality characterization of animal models is indispensable to delineate future strategies on clinical targets. To this end, the design of behavioral studies should place particular emphasis on quantitative, dimensional, and timespecific relations between face and construct validity. For instance, experiments on viral sequelae should take into account the behavioral and cognitive properties associated with the brain areas of viral tropism. Furthermore, the analysis of virus-induced disorders should be performed across different time points, to capture the progressive changes induced by the ongoing virus-mediated insult (through neurotoxic proteins and oxidative stress), immune responses (through cytokine release, microglial activation, etc.), neurodevelopmental processes, and neural compensatory mechanisms. For example, this conceptual framework has been employed to study the time-related effects of maternal influenza on cortical, hippocampal, and cerebellar morphogenesis [53, 96].

2.4. Experimental Design and Analysis: Tackling Problems of Inconsistency and Reproducibility. The experimental design related to the phenotyping of virus-induced sequelae is based on an extremely complex algorithm, which reflects a top-down progression from generic, multimodal paradigms towards increasingly specific tasks for the measurement of subtle traits. This approach is instrumental for the deconstructing strategy used for the identification of intermediate phenotypes.

As previously mentioned, the evolving state of the disorder dictates the need for a meticulous temporal documentation of the course of viral pathogenesis, which should be accomplished through periodic examinations of the infected rodents during their major developmental milestones. A general protocol for the assessment of neurobehavioral sequelae of experimental infections is outlined in Table 1. The phenotypic assessment should typically include auxological (size, body weight, etc.), physiological (heart rate, breathing frequency, body temperature, and food and water intake), and neurological parameters (posture, gait, motor coordination, reflex integrity, sensory perception, and pain sensitivity) [97]. Impairments in these functions may call for specific adjustments of the experimental design and testing procedure. For example, acoustic alterations may require paradigms relying on alternative sensory modalities, such as visual or tactile. Similarly, deficits in locomotor and cardiorespiratory functions may justify the selection of smaller arenas for behavioral testing and shorter experimental duration (to avoid fatigue). Finally, observations should include a brief ethological analysis of nesting, grooming, exploratory, and social behaviors, and so forth. This preliminary monitoring can be critical to refine hypotheses on potential behavioral impairments to test in the experimental phase.

The identification of intermediate behavioral phenotypes induced by early viral infection should be performed with a battery of subsequent tests. The operational procedure starts with the recognition of behavioral differences between virusinfected rodents and their controls in highly standardized paradigms, such as the open field. Alterations of the performance in this apparatus can inform on numerous components of behavioral and cognitive repertoire, such as locomotion, exploration, spontaneous activity, anxiety-like responses, behavioral rigidity, and memory (upon repeated exposure to the apparatus) [98]. The spectrum of openfield behaviors directs the selection of new paradigms to further qualify the nature of the observed variations. Subsequent adjustments towards progressively narrower targets reshape the architecture of the decisional flowchart and allow the detection of specific intermediate phenotypes. Testing conditions should always be optimized in view of the specific aspects of the experimentation, to capture potential variations with the highest sensitivity. In this context, environmental variations (such as light and noise level) in the experimental setting, as well as the employment of pharmacological agents can be extremely valuable to reveal hidden non-apparent traits and elucidate underlying neurobiological mechanisms.

As no single assay can completely model a psychiatric phenotype, conclusions based on a limited set of paradigms can prove detrimental to the analysis and lead to false positive (or negative) results. Instead, characterization of phenotypes should be based on a multimodal approach with complementary paradigms that can capture different facets of behavioral domains. More generally, experimental strategies aimed at the identification of virus-induced intermediate phenotypes should always be multifactorial and employ several complementary measures (including non-behavioral indices, such as neurochemical, electrophysiological, and physiological parameters). The documentation of coherent indications from multiple variables ensures the translational quality and the construct validity of the findings. 
TABLE 1: Experimental protocol for phenotyping of neurobehavioral sequelae of experimental infections in rodents.

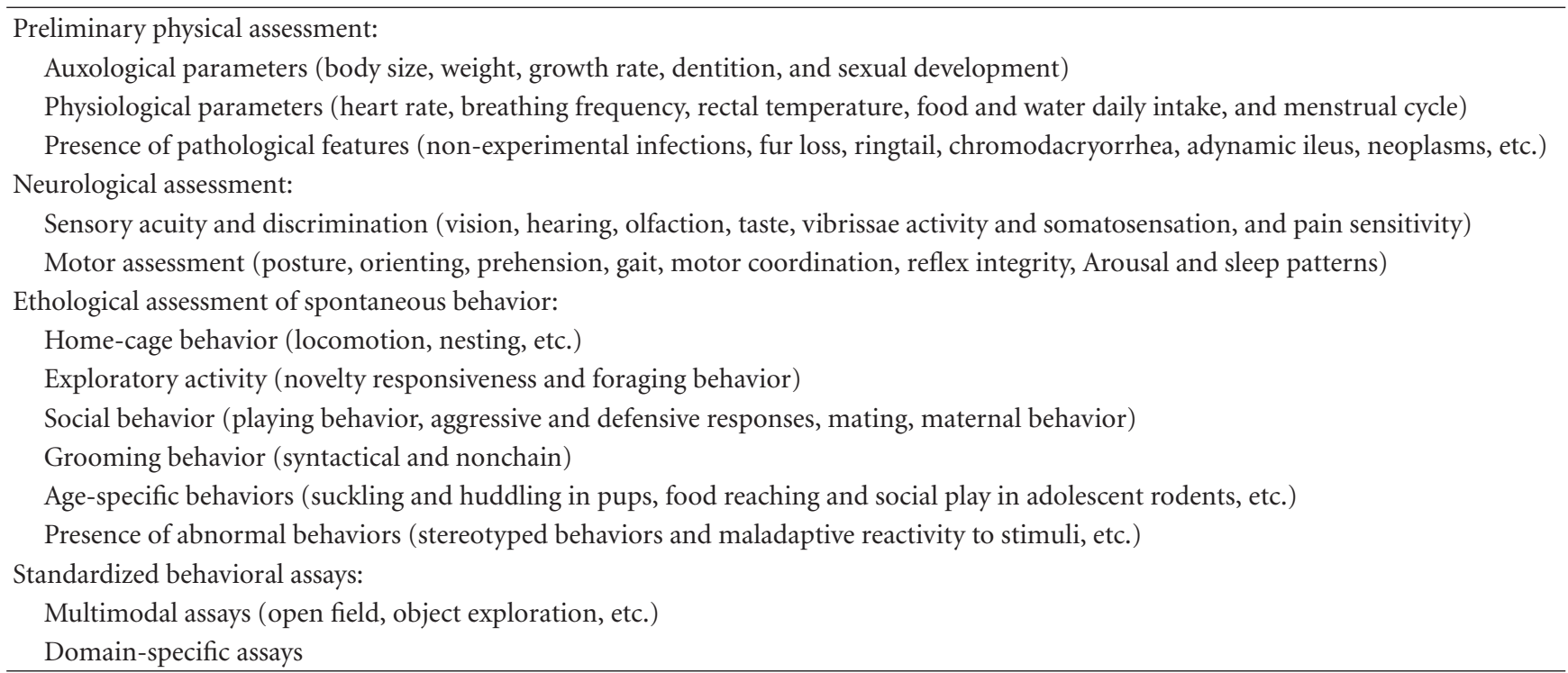

One of the most vexing and insidious aspects of behavioral research on virus-induced sequelae is the low reproducibility of findings across different laboratories and settings. This problem is generally ascribed to a large contingent of numerous unpredictable and irreducible environmental variables that cannot be fully accounted for in behavioral testing. Low reproducibility undermines the progress of behavioral neuroscience and the improvement of translational strategies, calling for urgent strategies to counter this problem.

One of the key strategies to maximize reproducibility in behavioral research is the standardization of experimental procedures. Nevertheless, this approach has been recently challenged [99], in light of the discrepant findings yielded by behavioral experiments performed with identical protocols in different laboratories. These authors have suggested an alternative approach to reduce intergroup variance, consisting in the heterogenization of experimental conditions.

Despite the persuasiveness of some of the arguments used to justify this strategy, standardization of certain conditions remains an essential requisite for some factors, such as gender and age. Furthermore, it is worth noting that intermediate phenotypes are defined as factors of vulnerability that are unraveled only in association with select genetic or environmental conditions; thus, insufficient standardization of conditions such as strain, husbandry, and testing conditions may temper the ability of a given paradigm to characterize and identify novel targets. This possibility is particularly concerning in reference to the analysis of virus-derived intermediate phenotypes. In fact, alterations induced by viral perturbation of a neural pathway may not result in full pathological processes, but may only confer a liability to a disorder. As this condition may be revealed only in presence of specific genetic or environmental factors, experimental heterogenization may impoverish the heuristic value of animal testing. These premises justify the need for stringent standardization throughout all the experimental phases of the study. In particular, extreme care should be exercised in the standardization of the following aspects:

(i) inoculation protocol: this standardization is critical to minimize apparent and predictable factors of variability, such as the characteristics of the host (strain, gender, age, etc.), the virus (strain, virulence, tropism), and the infection procedure (infectious dosage, regimen and route of administration). This latter concept is particularly important, as experimental infections are generally performed in utero or during critical stages of early postnatal neurodevelopment, which are characterized by the occurrence of numerous time-sensitive morphogenetic processes;

(ii) environmental conditions of husbandry and experimental testing: this prescription is essential to control (and preferably minimize) potential sources of stress. Indeed, non-viral stress interferes with the pathophysiological trajectory of virus-induced behavioral sequelae and is a major confounder in behavioral analysis. Stress-induced secretion of glucocorticoids leads to inhibition of cell immunity and reactivation of latent viruses [100-102]. Stress can also modulate immune functions through other hormones (including corticotrophin-releasing hormone, growth hormone, and prolactin) and neurotransmitters, such as catecholamines [103] and serotonin [104]. This aspect can become extremely critical with models of virus-induced sequelae that have been shown to alter monoamine signaling, such as influenza virus and BDV $[59,71,74,105,106]$.

The reliability and translational impact of the findings can also be enhanced with a thorough statistical approach, which should not only be restricted to comparisons between 
cases and controls based on ANOVA or its nonparametric counterparts. Temporal analysis of behavior can be fundamental to illustrate the progression of virus-induced alterations. Alternatively, correlational approaches can be employed to link particular behavioral outcomes to specific variations in antibody panel, neurotransmitter levels, morphology, electrophysiological alterations, drug sensitivity, or other changes in molecular substrates.

Data analysis can also be improved by the elimination of statistical artifacts, such as floor or ceiling effects, which can be overcome by the incorporation of alternative methods of analysis. Another typical example of artifact is offered by the "litter effect", defined as the tendency for littermates to exhibit similar responses to the same early insult. This potential pitfall can generate spurious statistical effects and should always be avoided by limiting the number of tested animals within each litter or by adjusting the statistical analysis of the behavioral data to account for kinship within the same treatment group.

Finally, a higher degree of replicability may be ensured by the adoption of different criteria for the rejection of null hypotheses. Indeed, the establishment of a $P$ level of .05 as a threshold for "statistical significance" has been extensively criticized [107, 108] and is accepted only on account of historical reasons [109]. However, the researcher should take into account that the employment of extremely stringent statistical conventions may lead to substantial drawbacks, such as the reduction of the positive predictive value and the heuristic validity of experimental procedures.

In conclusion, the design of any experiment aimed at behavioral phenotyping of virus-induced neurobehavioral sequelae should always take into account a number of key issues:

(i) definition of meaningful endpoints, based on intermediate phenotypes revealed by cross-species paradigms (testable in both humans and animals);

(ii) environmental standardization and elimination of exogenous stressors;

(iii) analysis at different time points, to capture the progression of viral pathogenesis;

(iv) correlational analysis between behavioral indices and other paradigms, to strengthen the construct validity of the animal model.

\section{Concluding Remarks and Future Directions}

Research on virus-induced neurobehavioral sequelae is advancing as new methodological developments in translational strategies and behavioral sciences set a promising scenario for future advancements. The field of phenotypic characterization is at a critical juncture and has recently witnessed the introduction of wide-range approaches, such as those afforded by transcriptomics, proteomics, metabonomics, and epigenetics. These techniques can provide a rich source of candidate traits for the identification of intermediate phenotypes $[110,111]$. Furthermore, these approaches can identify patterns of a specific "viral signature", which may help facilitate the diagnosis and the link with a viral etiology without requiring the analysis of viral material in numerous clinical specimens [112-114].

The establishment of a neurophenomic approach to psychiatric diagnosis and classification with extensive profiling of affective and cognitive traits through batteries of psychometric scales $[115,116]$ is bound to stimulate the development of parallel guidelines and establish a sound foundation for improvements in experimental design on animal models, with a particular emphasis on construct validity criteria. This perspective should strengthen our understanding of the neural mechanisms underlying the role of viruses in psychiatric disorders and accelerate the identification of therapeutic targets.

\section{References}

[1] K. A. Menninger, "Influenza and schizophrenia. An analysis of post-influenzal "dementia precox," as of 1918, and five years later further studies of the psychiatric aspects of influenza," American Journal of Psychiatry, vol. 82, no. 6, pp. 469-529, 1926.

[2] C. A. Kaufmann, J. R. Stevens, and E. F. Torrey, "1983 World Health Organization symposium on psychovirology," Archives of General Psychiatry, vol. 41, no. 12, pp. 1184-1185, 1984.

[3] F. L. Wilkie, K. Goodkin, M. H. Van Zuilen, M. D. Tyll, R. Lecusay, and T. Edwin, "Cognitive effects of HIV-1 infection," CNS Spectrums, vol. 5, no. 5, pp. 33-51, 2000.

[4] A. H. Mohammed, E. Norrby, and K. Kristensson, "Viruses and behavioural changes: a review of clinical and experimental findings," Reviews in the Neurosciences, vol. 4, no. 3, pp. 267-286, 1993.

[5] K. Tomonaga, "Virus-induced neurobehavioral disorders: mechanisms and implications," Trends in Molecular Medicine, vol. 10, no. 2, pp. 71-77, 2004.

[6] G. J. Atkins, I. M. Balluz, G. M. Glasgow, et al., "Analysis of the molecular basis of neuropathogenesis of RNA viruses in experimental animals: relevance for human disease?" Neuropathology and Applied Neurobiology, vol. 20, no. 2, pp. 91-102, 1994.

[7] M. Dalod, T. P. Salazar-Mather, L. Malmgaard, et al., "Interferon $\alpha / \beta$ and interleukin 12 responses to viral infections: pathways regulating dendritic cell cytokine expression in vivo," Journal of Experimental Medicine, vol. 195, no. 4, pp. 517-528, 2002.

[8] M. A. Muñoz-Fernández and M. Fresno, "The role of tumour necrosis factor, interleukin 6, interferon- $\gamma$ and inducible nitric oxide synthase in the development and pathology of the nervous system," Progress in Neurobiology, vol. 56, no. 3, pp. 307-340, 1998.

[9] J. S. Orange and C. A. Biron, "An absolute and restricted requirement for IL-12 in natural killer cell IFN- $\gamma$ production and antiviral defense: studies of natural killer and $\mathrm{T}$ cell responses in contrasting viral infections," Journal of Immunology, vol. 156, no. 3, pp. 1138-1142, 1996.

[10] T. P. Salazar-Mather, T. A. Hamilton, and C. A. Biron, "A chemokine-to-cytokine-to-chemokine cascade critical in antiviral defense," Journal of Clinical Investigation, vol. 105, no. 7, pp. 985-993, 2000.

[11] Marco Bortolato and Marylou V. Solbrig, "The price of seizure control: dynorphins in interictal and postictal 
psychosis," Psychiatry Research, vol. 151, no. 1-2, pp. 139143, 2007.

[12] G. R. DeLong, S. C. Bean, and F. R. Brown, "Acquired reversible autistic syndrome in acute encephalopathic illness in children," Archives of Neurology, vol. 38, no. 3, pp. 191-194, 1981.

[13] D. A. Lewis, "Retroviruses and the pathogenesis of schizophrenia," Proceedings of the National Academy of Sciences of the United States of America, vol. 98, no. 8, pp. 42934294, 2001.

[14] J. H. Boyd, A. E. Pulver, and W. Stewart, "Season of birth: schizophrenia and bipolar disorder," Schizophrenia bulletin, vol. 12, no. 2, pp. 173-186, 1986.

[15] E. H. Hare, J. S. Price, and E. Slater, "Schizophrenia and season of birth," British Journal of Psychiatry, vol. 120, no. 554, pp. 124-125, 1972.

[16] R. A. Machon, S. A. Mednick, and F. Schulsinger, "The interaction of seasonality, place of birth, genetic risk and subsequent schizophrenia in a high risk sample," British Journal of Psychiatry, vol. 143, no. 4, pp. 383-388, 1983.

[17] E. F. Torrey, B. B. Torrey, and M. R. Peterson, "Seasonality of schizophrenic births in the United States," Archives of General Psychiatry, vol. 34, no. 9, pp. 1065-1070, 1977.

[18] B. D. Bartlik, "Monthly variation in births of autistic children in North Carolina," Journal of the American Medical Women's Association, vol. 36, no. 12, pp. 363-368, 1981.

[19] E. Fombonne, "Season of birth and childhood psychosis," British Journal of Psychiatry, vol. 155, pp. 655-661, 1989.

[20] C. Gillberg, "Do children with autism have March birthdays?” Acta Psychiatrica Scandinavica, vol. 82, no. 2, pp. 152 156, 1990.

[21] M. M. Konstantareas, P. Hauser, C. Lennox, and S. Homatidis, "Season of birth in infantile autism," Child Psychiatry and Human Development, vol. 17, no. 1, pp. 53-65, 1986.

[22] Y. Tanoue, S. Oda, F. Asano, and K. Kawashima, "Epidemiology of infantile autism in southern Ibaraki, Japan: differences in prevalence in birth cohorts," Journal of Autism and Developmental Disorders, vol. 18, no. 2, pp. 155-166, 1988.

[23] D. J. Hart, R. G. Heath, F. J. Sautter, et al., "Antiretroviral antibodies: implications for schizophrenia, schizophrenia spectrum disorders, and bipolar disorder," Biological Psychiatry, vol. 45, no. 6, pp. 704-714, 1999.

[24] S. Srikanth, V. Ravi, K. S. Poornima, K. T. Shetty, B. N. Gangadhar, and N. Janakiramaiah, "Viral antibodies in recent onset, nonorganic psychoses: correspondence with symptomatic severity," Biological Psychiatry, vol. 36, no. 8, pp. 517-521, 1994.

[25] H. Terayama, Y. Nishino, M. Kishi, K. Ikuta, M. Itoh, and K. Iwahashi, "Detection of anti-Borna Disease Virus (BDV) antibodies from patients with schizophrenia and mood disorders in Japan," Psychiatry Research, vol. 120, no. 2, pp. 201-206, 2003.

[26] S. L. Buka, M. T. Tsuang, E. F. Torrey, M. A. Klebanoff, D. Bernstein, and R. H. Yolken, "Maternal infections and subsequent psychosis among offspring," Archives of General Psychiatry, vol. 58, no. 11, pp. 1032-1037, 2001.

[27] S. L. Buka, T. D. Cannon, E. F. Torrey, and R. H. Yolken, "Maternal exposure to herpes simplex virus and risk of psychosis among adult offspring," Biological Psychiatry, vol. 63, no. 8, pp. 809-815, 2008.

[28] F. Dickerson, B. Kirkpatrick, J. Boronow, C. Stallings, A. Origoni, and R. Yolken, "Deficit schizophrenia: association with serum antibodies to cytomegalovirus," Schizophrenia Bulletin, vol. 32, no. 2, pp. 396-400, 2006.

[29] J. J. Kim, B. H. Shirts, M. Dayal, et al., "Are exposure to cytomegalovirus and genetic variation on chromosome $6 \mathrm{p}$ joint risk factors for schizophrenia?" Annals of Medicine, vol. 39, no. 2, pp. 145-153, 2007.

[30] E. F. Torrey, M. F. Leweke, M. J. Schwarz, et al., "Cytomegalovirus and schizophrenia," CNS Drugs, vol. 20, no. 11, pp. 879-885, 2006.

[31] C. E. Barr, S. A. Mednick, and P. Munk-Jorgensen, "Exposure to influenza epidemics during gestation and adult schizophrenia. A 40-year study," Archives of General Psychiatry, vol. 47, no. 9, pp. 869-874, 1990.

[32] T. D. Cannon, S. A. Mednick, J. Parnas, F. Schulsinger, J. Praestholm, and A. Vestergaard, "Developmental brain abnormalities in the offspring of schizophrenic mothers. I. Contributions of genetic and perinatal factors," Archives of General Psychiatry, vol. 50, no. 7, pp. 551-564, 1993.

[33] S. A. Mednick, R. A. Machon, M. O. Huttunen, and D. Bonett, "Adult schizophrenia following prenatal exposure to an influenza epidemic," Archives of General Psychiatry, vol. 45, no. 2, pp. 189-192, 1988.

[34] E. O'Callaghan, P. Sham, N. Takei, G. Glover, and R. M. Murray, "Schizophrenia after prenatal exposure to 1957 A2 influenza epidemic," Lancet, vol. 337, no. 8752, pp. 12481250, 1991.

[35] N. Takei, P. Sham, E. O’Callaghan, G. K. Murray, G. Glover, and R. M. Murray, "Prenatal exposure to influenza and the development of schizophrenia: is the effect confined to females?" American Journal of Psychiatry, vol. 151, no. 1, pp. 117-119, 1994.

[36] H. Karlsson, S. Bachmann, J. Schröder, J. McArthur, E. F. Torrey, and R. H. Yolken, "Retroviral RNA identified in the cerebrospinal fluids and brains of individuals with schizophrenia," Proceedings of the National Academy of Sciences of the United States of America, vol. 98, no. 8, pp. 46344639, 2001.

[37] H. Perron, L. Mekaoui, C. Bernard, F. Veas, I. Stefas, and M. Leboyer, "Endogenous retrovirus type W GAG and envelope protein antigenemia in serum of schizophrenic patients," Biological Psychiatry, vol. 64, no. 12, pp. 1019-1023, 2008.

[38] B. Anlar, F. Oktem, and T. Torok, "Human parvovirus B19 antibodies in infantile autism," Journal of Child Neurology, vol. 9, no. 1, pp. 104-105, 1994.

[39] E. Y. Deykin and B. MacMahon, "Original contributions. Viral exposure and autism," American Journal of Epidemiology, vol. 109, no. 6, pp. 628-638, 1979.

[40] A. D. Allen, H. H. Fudenberg, and R. E. Allen, "Affective disorder and viral infections," Archives of General Psychiatry, vol. 44, no. 8, p. 760, 1987.

[41] L. E. DeLisi, J. S. Nurnberger, L. R. Goldin, S. SimmonsAlling, and E. S. Gershon, "Epstein-Barr virus and depression," Archives of General Psychiatry, vol. 43, no. 8, pp. 815816, 1986.

[42] E. F. Torrey, R. H. Yolken, and C. J. Winfrey, "Cytomegalovirus antibody in cerebrospinal fluid of schizophrenic patients detected by enzyme immunoassay," Science, vol. 216, no. 4548, pp. 892-894, 1982.

[43] J. G. Millichap, "Etiologic classification of attentiondeficit/hyperactivity disorder," Pediatrics, vol. 121, no. 2, pp. e358-e365, 2008.

[44] M. R. Weed and L. H. Gold, "Paradigms for behavioral assessment of of viral pathogenesis," Advances in Virus Research, vol. 56, pp. 583-626, 2001. 
[45] P. Willner, "Validation criteria for animal models of human mental disorders: learned helplessness as a paradigm case," Progress in Neuropsychopharmacology and Biological Psychiatry, vol. 10, no. 6, pp. 677-690, 1986.

[46] L. Asp, S. Beraki, K. Kristensson, S. O. Ögren, and H. Karlsson, "Neonatal infection with neurotropic influenza A virus affects working memory and expression of type III Nrg1 in adult mice," Brain, Behavior, and Immunity, vol. 23, no. 6, pp. 733-741, 2009.

[47] L. Asp, M. Holtze, S. B. Powell, H. Karlsson, and S. Erhardt, "Neonatal infection with neurotropic influenza A virus induces the kynurenine pathway in early life and disrupts sensorimotor gating in adult Tap1-/- mice," International Journal of Neuropsychopharmacology. In press.

[48] S. Beraki, F. Aronsson, H. Karlsson, S. O. Ögren, and K. Kristensson, "Influenza A virus infection causes alterations in expression of synaptic regulatory genes combined with changes in cognitive and emotional behaviors in mice," Molecular Psychiatry, vol. 10, no. 3, pp. 299-308, 2005.

[49] C. A. Conn, J. L. McClellan, H. F. Maassab, C. W. Smitka, J. A. Majde, and M. J. Kluger, "Cytokines and the acute phase response to influenza virus in mice," American Journal of Physiology, vol. 268, no. 1, pp. R78-R84, 1995.

[50] L. Shi, S. H. Fatemi, R. W. Sidwell, and P. H. Patterson, "Maternal influenza infection causes marked behavioral and pharmacological changes in the offspring," Journal of Neuroscience, vol. 23, no. 1, pp. 297-302, 2003.

[51] L. A. Toth, J. E. Rehg, and R. G. Webster, "Strain differences in sleep and other pathophysiological sequelae of influenza virus infection in naive and immunized mice," Journal of Neuroimmunology, vol. 58, no. 1, pp. 89-99, 1995.

[52] S. H. Fatemi, E. S. Emamian, R. W. Sidwell, et al., "Human influenza viral infection in utero alters glial fibrillary acidic protein immunoreactivity in the developing brains of neonatal mice," Molecular Psychiatry, vol. 7, no. 6, pp. 633-640, 2002.

[53] S. H. Fatemi, J. Earle, R. Kanodia, et al., "Prenatal viral infection leads to pyramidal cell atrophy and macrocephaly in adulthood: implications for genesis of autism and schizophrenia," Cellular and Molecular Neurobiology, vol. 22, no. 1, pp. 25-33, 2002.

[54] D. Cotter, N. Takei, M. Farrell, et al., "Does prenatal exposure to influenza in mice induce pyramidal cell disarray in the dorsal hippocampus?" Schizophrenia Research, vol. 16, no. 3, pp. 233-241, 1995.

[55] S. H. Fatemi, E. S. Emamian, D. Kist, et al., "Defective corticogenesis and reduction in Reelin immunoreactivity in cortex and hippocampus of prenatally infected neonatal mice," Molecular Psychiatry, vol. 4, no. 2, pp. 145-154, 1999.

[56] S. H. Fatemi, R. Sohr, G. Juckel, et al., "Maternal infection leads to abnormal gene regulation and brain atrophy in mouse offspring: implications for genesis of neurodevelopmental disorders," Schizophrenia Research, vol. 99, no. 1-3, pp. 56-70, 2008.

[57] S. H. Fatemi, T. D. Folsom, T. J. Reutiman, et al., "Abnormal expression of myelination genes and alterations in white matter fractional anisotropy following prenatal viral influenza infection at E16 in mice," Schizophrenia Research, vol. 112, no. 1-3, pp. 46-53, 2009.

[58] L. Shi, S. E. P. Smith, N. Malkova, D. Tse, Y. Su, and P. H. Patterson, "Activation of the maternal immune system alters cerebellar development in the offspring," Brain, Behavior, and Immunity, vol. 23, no. 1, pp. 116-123, 2009.
[59] C. Winter, T. J. Reutiman, T. D. Folsom, et al., "Dopamine and serotonin levels following prenatal viral infection in mouse-implications for psychiatric disorders such as schizophrenia and autism," European Neuropsychopharmacology, vol. 18, no. 10, pp. 712-716, 2008.

[60] David R. Beers, Jenny S. Henkel, Raymond P. Kesner, and William G. Stroop, "Spatial recognition memory deficits without notable CNS pathology in rats following herpes simplex encephalitis," Journal of the Neurological Sciences, vol. 131, no. 2, pp. 119-127, 1995.

[61] L. S. Crnic and L. I. Pizer, "Behavioral effects of neonatal herpes simplex type 1 infection of mice," Neurotoxicology and Teratology, vol. 10, no. 4, pp. 381-386, 1988.

[62] J. A. Engel, J. Zhang, T. Bergström, et al., "Neonatal herpes simplex virus type 1 brain infection affects the development of sensorimotor gating in rats," Brain Research, vol. 863, no. 1-2, pp. 233-240, 2000.

[63] D. J. McFarland and J. Hotchin, "Host genetics and the behavioral sequelae to herpes encephalitis in mice," Physiology and Behavior, vol. 30, no. 6, pp. 881-884, 1983.

[64] E. Lycke and B. E. Roos, "Virus infection in infant mice causing persistent impairment of turnover of brain catecholamines," Journal of the Neurological Sciences, vol. 26, no. 1, pp. 49-60, 1975.

[65] H. M. Wu, C. C. Huang, S. H. Chen, et al., "Herpes simplex virus type 1 inoculation enhances hippocampal excitability and seizure susceptibility in mice," European Journal of Neuroscience, vol. 18, no. 12, pp. 3294-3304, 2003.

[66] C. Belzung, "The genetic basis of the pharmacological effects of anxiolytics: A review based on rodent models," Behavioural Pharmacology, vol. 12, no. 6-7, pp. 451-460, 2001.

[67] A. Markou, C. Chiamulera, M. A. Geyer, M. Tricklebank, and T. Steckler, "Removing obstacles in neuroscience drug discovery: the future path for animal models," Neuropsychopharmacology, vol. 34, no. 1, pp. 74-89, 2009.

[68] J. M. Pyper, "Does Borna disease virus infect humans?" Nature Medicine, vol. 1, no. 3, pp. 209-210, 1995.

[69] K. Lieb and P. Staeheli, "Borna disease virus-does it infect humans and cause psychiatric disorders?" Journal of Clinical Virology, vol. 21, no. 2, pp. 119-127, 2001.

[70] T. Briese, M. Hornig, and W. I. Lipkin, "Bornavirus immunopathogenesis in rodents: models for human neurological diseases," Journal of NeuroVirology, vol. 5, no. 6, pp. 604-612, 1999.

[71] M. V. Solbrig, G. F. Koob, J. N. Joyce, and W. I. Lipkin, “A neural substrate of hyperactivity in Borna disease: changes in brain dopamine receptors," Virology, vol. 222, no. 2, pp. 332338, 1996.

[72] A. Hans, J. J. Bajramovic, S. Syan, et al., "Persistent, noncytolytic infection of neurons by Borna disease virus interferes with ERK 1/2 signaling and abrogates BDNFinduced synaptogenesis," The FASEB Journal, vol. 18, no. 7, pp. 863-865, 2004.

[73] M. Hornig, H. Weissenböck, N. Horscroft, and W. I. Lipkin, "An infection-based model of neurodevelopmental damage," Proceedings of the National Academy of Sciences of the United States of America, vol. 96, no. 21, pp. 12102-12107, 1999.

[74] M. V. Solbrig, G. F. Koob, J. H. Fallon, and W. I. Lipkin, "Tardive dyskinetic syndrome in rats infected with Borna disease virus," Neurobiology of Disease, vol. 1, no. 3, pp. 111119, 1994.

[75] M. V. Solbrig, G. F. Koob, J. H. Fallon, S. Reid, and W. I. Lipkin, "Prefrontal cortex dysfunction in Borna disease virus 
(BDV)-infected rats," Biological Psychiatry, vol. 40, no. 7, pp. 629-636, 1996.

[76] M. V. Solbrig, G. F. Koob, L. H. Parsons, et al., "Neurotrophic factor expression after CNS viral injury produces enhanced sensitivity to psychostimulants: potential mechanism for addiction vulnerability," The Journal of Neuroscience, vol. 20, no. 21, p. RC104, 2000.

[77] S. E. Hyman, "Can neuroscience be integrated into the DSMV?” Nature Reviews Neuroscience, vol. 8, no. 9, pp. 725-732, 2007.

[78] Y. Lecrubier, "Refinement of diagnosis and disease classification in psychiatry," European Archives of Psychiatry and Clinical Neuroscience, vol. 258, no. 1, pp. 6-11, 2008.

[79] K. A. Phillips, S. Van Bebber, and A. M. Issa, "Diagnostics and biomarker development: priming the pipeline," Nature Reviews Drug Discovery, vol. 5, no. 6, pp. 463-469, 2006.

[80] M. Leboyer, F. Bellivier, R. Jouvent, M. Nosten-Bertrand, J. Mallet, and D. Pauls, "Psychiatric genetics: search for phenotypes," Trends in Neurosciences, vol. 21, no. 3, pp. 102105, 1998.

[81] I. I. Gottesman and J. Shields, "Genetic theorizing and schizophrenia," British Journal of Psychiatry, vol. 122, no. 566, pp. 15-30, 1973.

[82] T. D. Gould and I. I. Gottesman, "Psychiatric endophenotypes and the development of valid animal models," Genes, Brain and Behavior, vol. 5, no. 2, pp. 113-119, 2006.

[83] B. Arts, N. Jabben, L. Krabbendam, and J. Van Os, "Meta-analyses of cognitive functioning in euthymic bipolar patients and their first-degree relatives," Psychological Medicine, vol. 38, no. 6, pp. 771-785, 2008.

[84] B. Viswanath, Y. C. J. Reddy, K. J. Kumar, T. Kandavel, and C. R. Chandrashekar, "Cognitive endophenotypes in OCD: a study of unaffected siblings of probands with familial OCD," Progress in Neuro-Psychopharmacology and Biological Psychiatry, vol. 33, no. 4, pp. 610-615, 2009.

[85] H. S. Hoffman and J. R. Ison, "Reflex modification in the domain of startle: I. Some empirical findings and their implications for how the nervous system processes sensory input," Psychological Review, vol. 87, no. 2, pp. 175-189, 1980.

[86] D. L. Braff, C. Grillon, and M. A. Geyer, "Gating and habituation of the startle reflex in schizophrenic patients," Archives of General Psychiatry, vol. 49, no. 3, pp. 206-215, 1992.

[87] D. L. Braff, M. A. Geyer, and N. R. Swerdlow, "Human studies of prepulse inhibition of startle: Normal subjects, patient groups, and pharmacological studies," Psychopharmacology, vol. 156, no. 2-3, pp. 234-258, 2001.

[88] K. S. Cadenhead, N. R. Swerdlow, K. M. Shafer, M. Diaz, and D. L. Braff, "Modulation of the startle response and startle laterality in relatives of schizophrenic patients and in subjects with schizotypal personality disorder: evidence of inhibitory deficits," American Journal of Psychiatry, vol. 157, no. 10, pp. 1660-1668, 2000.

[89] D. L. Braff, R. Freedman, N. J. Schork, and I. I. Gottesman, "Deconstructing schizophrenia: an overview of the use of endophenotypes in order to understand a complex disorder," Schizophrenia Bulletin, vol. 33, no. 1, pp. 21-32, 2007.

[90] F. X. Castellanos and R. Tannock, "Neuroscience of attentiondeficit/hyperactivity disorder: the search for endophenotypes," Nature Reviews Neuroscience, vol. 3, no. 8, pp. 617$628,2002$.

[91] R. H. Lenox, T. D. Gould, and H. K. Manji, "Endophenotypes in bipolar disorder," American Journal of Medical Genetics, vol. 114, no. 4, pp. 391-406, 2002.
[92] K. R. Merikangas, A. Chakravarti, S. O. Moldin, H. Araj, J. C. Blangero, and M. Burmeister, "Future of genetics of mood disorders research," Biological Psychiatry, vol. 52, no. 6, pp. 457-477, 2002.

[93] D. H. Skuse, "Endophenotypes and child psychiatry," British Journal of Psychiatry, vol. 178, pp. 395-396, 2001.

[94] C. E. Bearden, A. J. Jasinska, and N. B. Freimer, "Methodological issues in molecular genetic studies of mental disorders," Annual Review of Clinical Psychology, vol. 5, pp. 49-69, 2009.

[95] M. Rutter, "Biological implications of gene-environment interaction," Journal of Abnormal Child Psychology, vol. 36, no. 7, pp. 969-975, 2008.

[96] S. H. Fatemi, M. Araghi-Niknam, J. A. Laurence, J. M. Stary, S. Lee, and R. W. Sidwell, "Glial fibrillary acidic protein and glutamic acid decarboxylase 65 and $67 \mathrm{kDa}$ proteins are increased in brains of neonatal BALB/c mice following viral infection in utero," Schizophrenia Research, vol. 69, no. 1, pp. 121-123, 2004.

[97] J. N. Crawley, "Behavioral phenotyping strategies for mutant mice," Neuron, vol. 57, no. 6, pp. 809-818, 2008.

[98] C. Belzung, "Measuring rodent exploratory behavior," in Handbook of Molecular-Genetic Techniques for Brain and Behavior Research, vol. 13 of Techniques in the Behavioral and Neural Sciences, pp. 738-749, Elsevier, Amsterdam, The Netherlands, 1999.

[99] S. H. Richter, J. P. Garner, and H. Würbel, "Environmental standardization: cure or cause of poor reproducibility in animal experiments?" Nature Methods, vol. 6, no. 4, pp. 257261, 2009.

[100] J. L. Lathey and S. A. Spector, "Unrestricted replication of human cytomegalovirus in hydrocortisone-treated macrophages," Journal of Virology, vol. 65, no. 11, pp. 63716375, 1991.

[101] P. D. Markham, S. Z. Salahuddin, and K. Veren, "Hydrocortisone and some other hormones enhance the expression of HTLV-III," International Journal of Cancer, vol. 37, no. 1, pp. 67-72, 1986.

[102] V. Schuster, E. Dohrmann, and H. W. Kreth, "Epstein-Barr virus-associated lymphoproliferative syndromes: studies in two European families," Cancer Detection and Prevention, vol. 15, no. 1, pp. 65-67, 1991.

[103] C. W. Cotman, R. E. Brinton, A. Galaburda, B. McEwen, and D. M. Schneider, Eds., The Neuro-Immuno-Endocrine Connection, Raven Press, New York, NY, USA, 1987.

[104] F. Chaouloff, "Serotonin, stress and corticoids," Journal of Psychopharmacology, vol. 14, no. 2, pp. 139-151, 2000.

[105] D. M. Dietz, M. W. Vogel, S. A. Rubin, T. H. Moran, K. M. Carbone, and M. V. Pletnikov, "Developmental alterations in serotoninergic neurotransmission in Borna disease virus (BDV)-infected rats: a multidisciplinary analysis," Journal of NeuroVirology, vol. 10, no. 5, pp. 267-277, 2004.

[106] M. V. Pletnikov, S. A. Rubin, G. J. Schwartz, K. M. Carbone, and T. H. Moran, "Effects of neonatal rat Borna disease virus (BDV) infection on the postnatal development of the brain monoaminergic systems," Developmental Brain Research, vol. 119, no. 2, pp. 179-185, 2000.

[107] S. N. Goodman, "Toward evidence-based medical statistics. 1: the P value fallacy," Annals of Internal Medicine, vol. 130, no. 12, pp. 995-1004, 1999.

[108] S. N. Goodman, "Of p-values and bayes: p modest proposal," Epidemiology, vol. 12, no. 3, pp. 295-297, 2001. 
[109] M. Cowles and C. Davis, "On the origins of the .05 level of statistical significance," American Psychologist, vol. 37, no. 5, pp. 553-558, 1982.

[110] I. Glegling, A. M. Hartmann, J. Genius, J. Benninghoff, H. J. Möller, and D. Rujescu, "Systems biology and complex neurobehavioral traits," Pharmacopsychiatry, vol. 41, no. 1, pp. S32-S36, 2008.

[111] H. H. H. Göring, A. G. Comuzzie, M. C. Mahaney, et al., "Discovery of expression QTLs using large-scale transcriptional profiling in human lymphocytes," Nature Genetics, vol. 39, no. 10, pp. 1208-1216, 2007.

[112] G. Pendyala, E. J. Want, W. Webb, G. Siuzdak, and H. S. Fox, "Biomarkers for neuroAIDS: the widening scope of metabolomics," Journal of Neuroimmune Pharmacology, vol. 2, no. 1, pp. 72-80, 2007.

[113] G. Pendyala, S. A. Trauger, E. Kalisiak, R. J. Ellis, G. Siuzdak, and H. S. Fox, "Cerebrospinal fluid proteomics reveals potential pathogenic changes in the brains of SIV-infected monkeys," Journal of Proteome Research, vol. 8, no. 5, pp. 2253-2260, 2009.

[114] B. L. Williams, K. Yaddanapudi, C. M. Kirk, A. Soman, M. Hornig, and W. I. Lipkin, "Metallothioneins and zinc dysregulation contribute to neurodevelopmental damage in a model of perinatal viral infection," Brain Pathology, vol. 16, no. 1, pp. 1-14, 2006.

[115] A. B. Niculescu 3rd and J. R. Kelsoe, "The human genome: genetic testing and animal models," American Journal of Psychiatry, vol. 158, no. 10, p. 1587, 2001.

[116] A. B. Niculescu, L. L. Lulow, C. A. Ogden, et al., "PhenoChipping of psychotic disorders: a novel approach for deconstructing and quantitating psychiatric phenotypes," American Journal of Medical Genetics B, vol. 141, no. 6, pp. 653-662, 2006. 


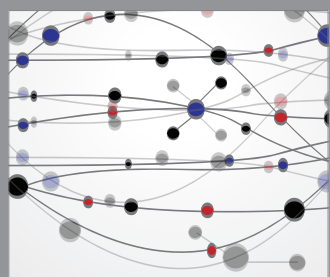

The Scientific World Journal
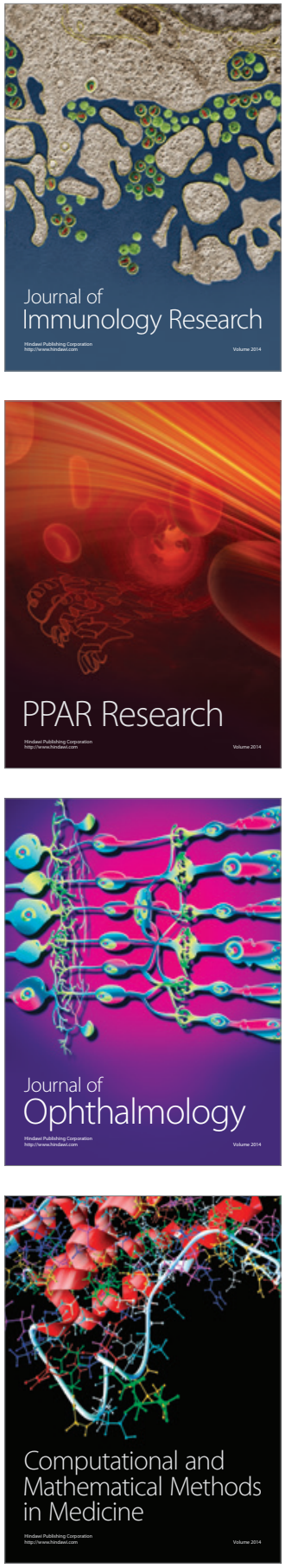

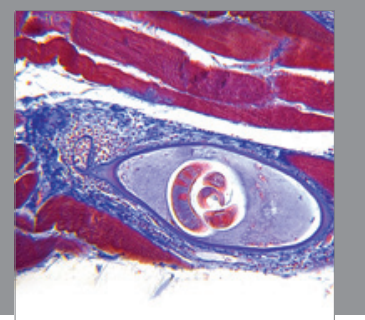

Gastroenterology

Research and Practice
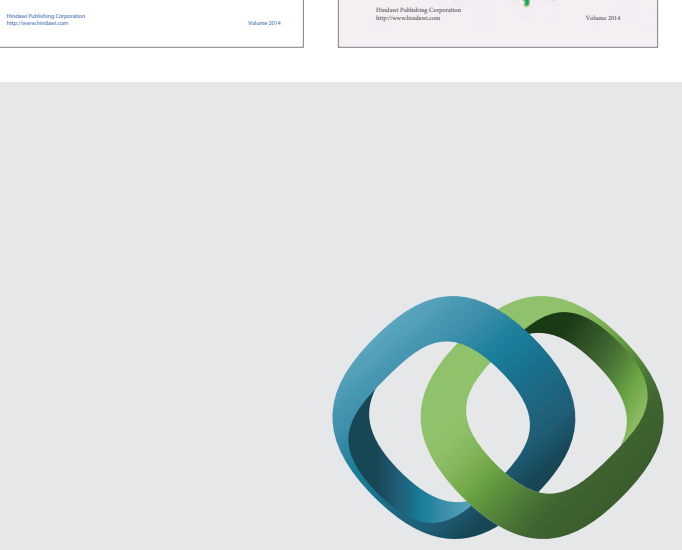

\section{Hindawi}

Submit your manuscripts at

http://www.hindawi.com
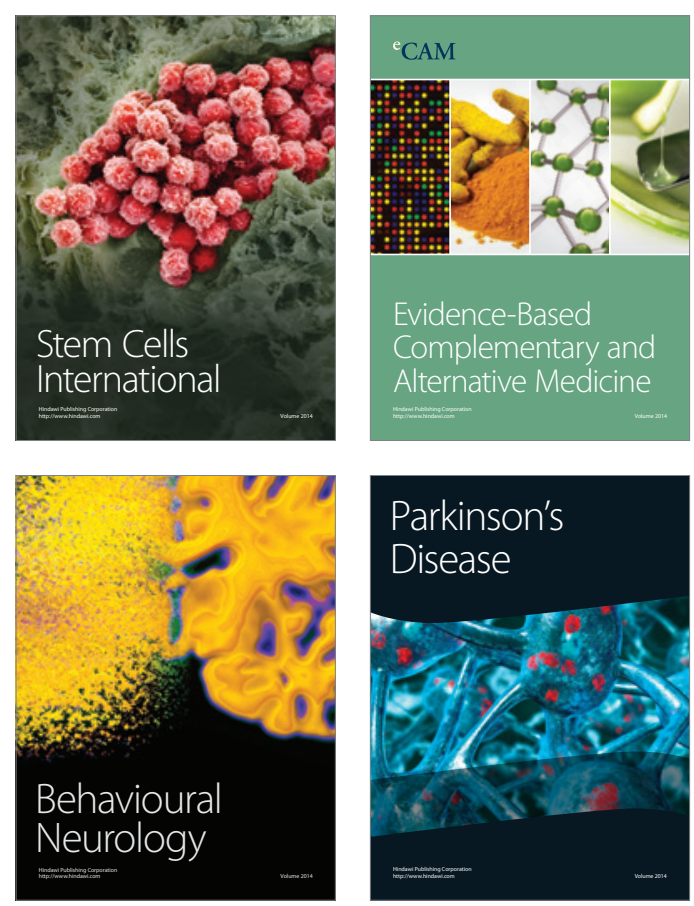

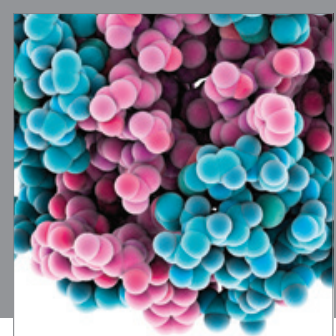

Journal of
Diabetes Research

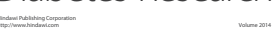

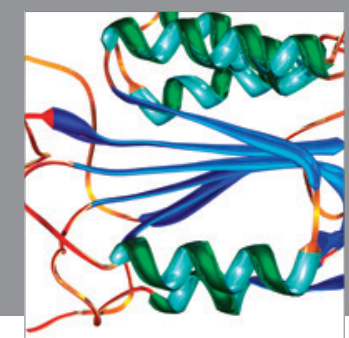

Disease Markers
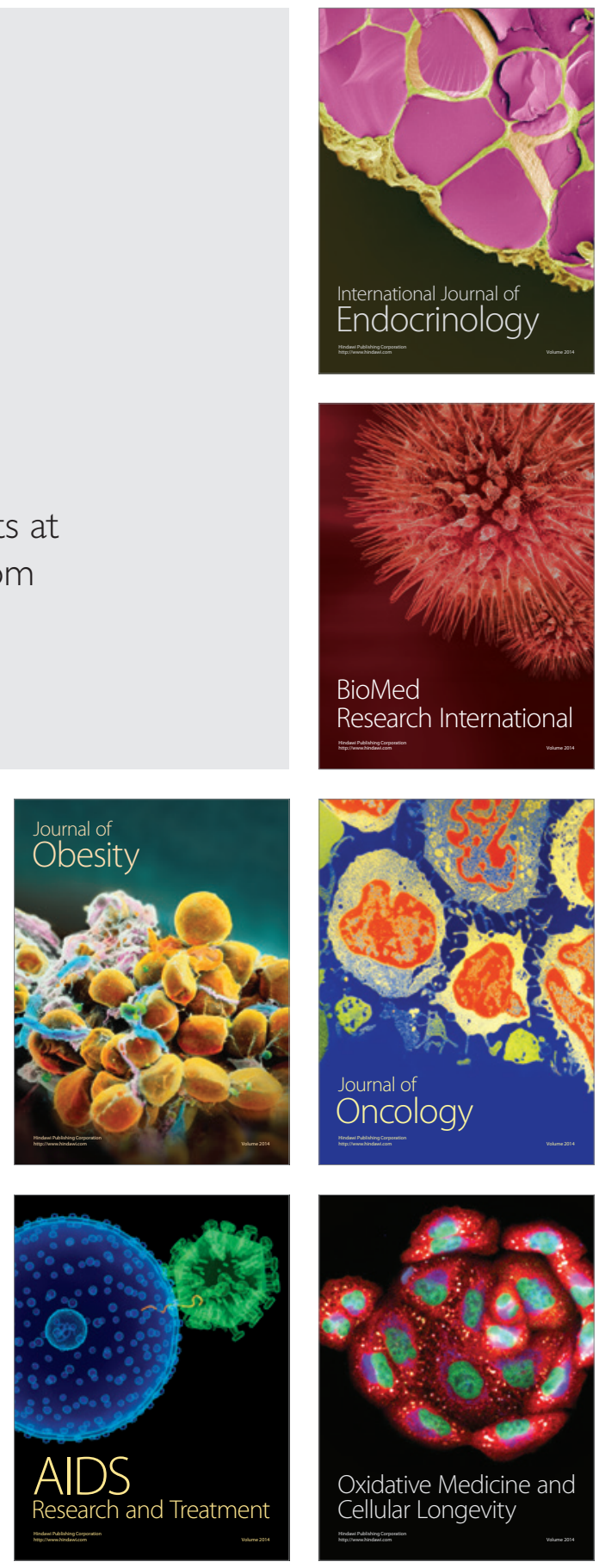